\title{
Non-intubated video-assisted left cardiac sympathetic denervation with high-flow nasal oxygen for the treatment of hereditary ventricular arrhythmias
}

\author{
A CASE REPORT
}

Yu-Ting Hsu, MD1, Chien-Kun Ting, MD, PhD'1, Po-Kuei Hsu, MD², Wei-Nung Teng, MD1,

Fa-Po Chung, MD ${ }^{3}$, Mei-Yung Tsou, MD, PhD ${ }^{1}$

1 Department of Anesthesiology, Taipei Veterans General Hospital, Taiwan

2 Department of Surgery, Taipei Veterans General Hospital, Taiwan

3 Division of Cardiology, Department of Medicine, Taipei Veterans General Hospital, Taiwan

\section{Background}

Left cardiac sympathetic denervation (LCSD) is an effective second-line therapy for catecholaminergic polymorphic ventricular tachycardia (CPVT).

Non-intubated video-assisted thoracic surgery is growing in popularity owing to sparing from side effects of general anesthesia and mechanical ventilation. Here, we reported two cases of non-intubated video-assisted LCSD with high-flow nasal cannula (HFNC; Optiflow, Fisher \& Paykel Healthcare).

\section{Case Report}

The first case is a 20-year-old man, and the second case is a 34-year-old man. Both patients were diagnosed with CPVT and suffered from frequent shocks from implanted ICD. Arriving our operating room, the patients were sedated with intravenous midazolam and alfentanil. Thoracic epidural catheter was placed and tested for efficacy. Single bolus injection with bupivacaine and fentanyl was given intra-epidurally. After placing the patients in decubitus position, propofol was given by target controlled infusion. Oxygen flow was increased to $50 \mathrm{~L} / \mathrm{min}$ after the patients were unresponsive.

No episodes of apnea or desaturation were observed during the procedures. Both patients' vital signs remained stable. There were no intra-operative episodes of ventricular arrhythmias. The operations were performed smoothly.

\section{Discussion}

Anesthetic management for patients undergoing LCSD could be challenging. In our experience, the combination of thoracic epidural anesthesia and target controlled propofol infusion offered reliable analgesia and unconsciousness with minimal changes on patients' hemodynamic states. The patients reserved spontaneous respiration during the whole surgeries, and excellent oxygenation was provided by HFNC, which is a promising new oxygenation technique. Its applications in anesthesia includes improving safety in awake craniotomy ${ }^{1}$, eyes or dental procedures, and also in nonintubated video-assisted thoracic surgery.

We demonstrated the safe and successful anesthesia using HFNC in non-intubated video-assisted LCSD for treatment of CPVT. The non-intubated technique avoids abrupt changes in hemodynamic status and allows faster recovery. And the HFNC ensures oxygenation during the operations.

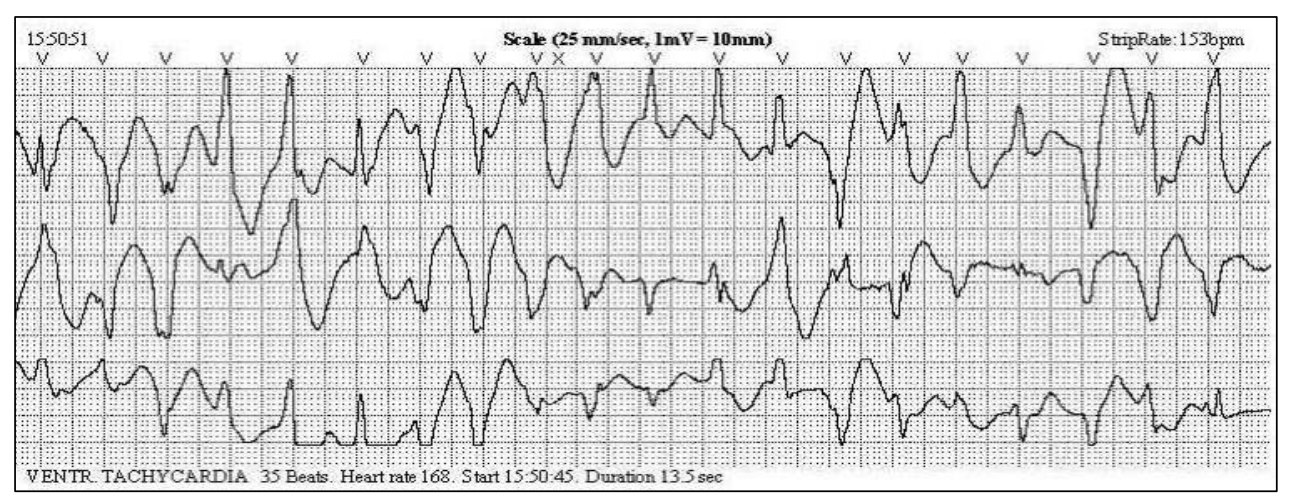

Fig. 1. 24-hours Holter's scan of the second case captured episodes of short-run ventricular tachycardia. The diagnosis of catecholaminergic polymorphic ventricular tachycardia was made through further electrophysiological study.

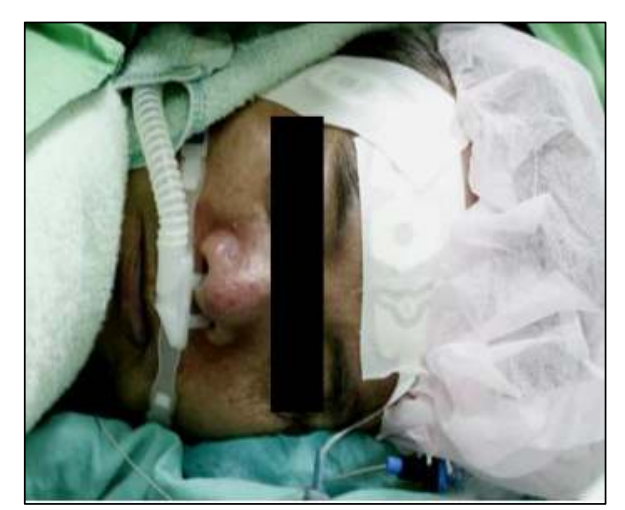

Fig. 2. The non-intubated technique was achieved with high-flow nasal cannula. 